\title{
Micro-Electro-Mechanical Systems United Constrained Model Construction and Application
}

\author{
Gong youping, Chen guojin, Li Zhihua \\ School of Mechanical Engineering Hangzhou Dianzi University Hangzhou, China \\ gypcad@163.com, chenguojin@163.com
}

\begin{abstract}
Micro-Electro-Mechanical Systems (MEMS) are the integration of mechanical elements, sensors, actuators, and electronics at the micron-scale or even at nano-scale through micro fabrication technology, which involves the mechanical, electronic, fluid, thermal, optical, magnetic and other disciplines, how to quickly and accurately modeling and simulating the MEMS has been a big problem, there are so many methods have been put forward to solve the problems:(1) Numerical Simulation Method (2) Signal Flow Analysis Method, (3) Nodal Analysis Method,.(4) Bond Graph Method With the development of computer technology, the united model construction language have been used in some areas, such as engineering mechanical, energy power fielded, also, this method can solve the MEMS simulation and optimizing problem, the paper mainly put forward to the uniform model construction simulation method. The method adopt uniform constraints to describe MEMS multi-fields simulation model, firstly construct MEMS model library which includes structure, electricty,magnetic,thermal,and fluid elements in multi-field uniform language modelica, through these elements, MEMS physics simulation model can be directly constructed, after model construction, some prosperities of the MEMS devices can be simulated.

Index Terms - MEMS, Multi-fields Simulation, modelica
\end{abstract}

\section{Introduction}

The scholars of the United States, Europe, Japan and other countries have realized the successful industrialization of micro-electro-mechanical system (MEMS) and occupying the market largely depend on how to use the computer aided design, analysis and manufacturing methods (CAD/CAE/CAM) effectively. Currently, in the study of MEMS, the development level of computer aided tools far behind the frontiers of the pace, most MEMS devices are designed by the analysis tools which have the similar functions and can not predict the execution situation of MEMS accurately. Therefore, we always use test troubleshooting methods, which often requires repeated trials to finalize the device equipment to meet the specific environment. For the development of MEMS devices and systems for commercial products, this backward design method has a long design cycle, low efficiency, high cost and impractical. Thus, there is an urgent need of advanced modeling tools and simulation methods for MEMS design. The multi-disciplinary nature of MEMS involves the complex coupling issues among the electric, mechanical, magnetic, thermal, fluid and optical physical multi-energy domain, and its integrated development trend needs a system simulation method of the device or system and circuit. At present, the simulation method that mainly used in MEMS, including numerical simulation, hardware description language method and the unified model of multi-domain simulation method, in which the hardware description language method develops into the signal flow analysis, nodal analysis, the bond graph method[1-3].

\section{II . Traditional simulation method in MEMS}

\section{A. Numerical Simulation Method}

Numerical simulation method includes the difference, finite element method, the boundary element, the finite volume method and other methods, the finite element method is the commonly used method, when we do the multi-energy domain coupling analysis of MEMS, we should seek the appropriate algorithm for the characteristics of each domain, and solve the coupling issues between different domains. Numerical simulation using ANSYS and other finite element software's or Intellisuite and other dedicated MEMS CAD software's to design and analyze MEMS structures. This method requires a pre-set of the structural parameters to make the model and simulation, and then amend the original model according to the results of the simulation analysis until we get the satisfactory results. This simulation method has a greater progress than repeating trials, it reduce the costs and shorten the design cycle to a certain extent. Some well-known finite element software such as ANSYS has successfully introduced a coupling field calculation method to solve a variety of coupling issues $[4,5]$. Li jun and others have made a finite element modeling and analysis of the electrostatic micropump[6]. Ji kexing and others have used the finite element method to study the influence of residual stress on the structural design of electrostatic feedback MEMS sensor with high temperature shear stress [7]. Field-based analysis of large-scale numerical simulation for different energy domain analysis needs to mesh, needs to achieve automatic mesh division which is very difficult, and the calculation method has not reached a mature stage. The calculation is huge and there is a lack of efficient method of calculating, so we can not achieve the simulation of system-level and circuit integration.

\section{B. Signal Flow Analysis Method}

The signal flow analysis is also known as black-box analysis. The basic idea is to establish on the basis of numerical analysis to different energy domains of MEMS coupling field, selected a few parameters to describe the energy of the system, reduce the system's degrees of freedom and do not care about the local structure and characteristics of the system. Then, the system coupling field can be expressed 
by a black-box which has a few parameters. Generally we select the vibration modal of the sporty structures as the basic variables set, we use the generalized coordinates for the coefficient of linear superposition to get the approximate deformation of the sport structures, establishing the Lagrange equation of the system, converting it for the hardware description language model and having a simulation in circuit of the MEMS, then, the simulation time of system can be greatly reduced. This method can obtain a high precision, and applies to any complex system [8,9].

\section{Nodal Analysis Method}

Using the node analysis to system modeling are in the form of the system unit geometry size and material constants components for simulation parameters, its ideology is similar to circuit analysis, considering the system is composed by the sub-structural units of the same energy domain or different energy domains, these component units with lumped parameter model description, interconnected by the nodes which have the same energy domain nature, equivalent to the circuit resistance, capacitance and so on. After converting it into hardware description language model, then link the units together, form a network topology structure, build the whole differential equations of system and implement the system simulation in the software of EDA. The advantage of the method is fully integrated with IC CAD tools, using the same software tools to solve MEMS and IC design problems at the same time, applying to the structures of lumped mass and stiffness can be decomposed, it is always used for the small displacement and small signal occasions. Jan E.Vandemeer has proposed nodal analysis for MEMS simulation and design [10], Q.Jing improved the nodal analysis method, and applied it into the MEMS parametric analysis [11]. Yuan weizheng used the improved nodal analysis method to solve the MEMS issues [12].

\section{Bond Graph Method}

As a powerful theoretical tool for the interaction between the different energy domain systems, the bond graph method makes the dynamic system as a system determined by the state variables, its relationship between the state variables and other system variables is established by a set of ordinary differential equations and algebraic equations. By using the bond graph language to describe the system model, different systems can exchange energy to achieve interaction. But the theory of the method is abstract, difficult to understand, incompatible with the electric network system simulator, it is difficult to achieve system-level simulation and optimization.

\section{Mems Multi - Domain Uniform Modeling And Simulation}

To early design of MEMS, we often considered the MEMS device structure itself more, and did less departure from a multidisciplinary point of view of technology, structure, fluid circuit, error to make the optimal design of the MEMS system. Even though the device itself achieves the best performance, once it is processed and used in conjunction with other peripheral subsystems, it can not achieve the optimal overall properties of the system, thereby increasing the number of iterations of the design, resulting in the improvement of product development costs. Therefore, it is necessary to study the MEMS simulation and optimization of design from the point of view of system, but there is still a lack of effective design methods to solve system design and optimization of MEMS. The method based on unified modeling language using a unified approach to describe the system components in different areas, it can achieve full realization of the seamless integration and data exchange between different areas of the model. The intrinsic component connection mechanism based on a unified language realizes the model composition and multi-domain integration, it can model and simulate all the problem which can be described by differential equations or algebraic equations.

The unified modeling studies of multi-domain physical system originated in the first object-oriented simulation modeling language Dymola by Elmqvist in 1978[13]. A series of object-oriented and equation-based simulation modeling language appeared in the later, for example, Omola, ASCEND, GPROMS, NMF, ObjectMath, Smile and so on[14]. Faced with the compatibility and standardization issues of many languages coexist, some experts and scholars in the simulation community in Europe had introduced a new generation of statement language Modelica on the basis of the previously summarized and unified a variety of modeling languages in 1997[15]. Currently, the modeling based on the Modelica language has a wide range of applications in the automotive and electric vehicles, hydraulic systems, mechanical multibody system, thermal power system, power systems, electromechanical systems, chemical systems, and hardware in loop simulation, discrete event systems and other systems of processes. The simulation application based on Modelica has been started in our country. For example, the simulation analysis of gas turbines, switched reluctance motor, synchronous motor, heat engine, engine, mechanical gearbox and turbofan engine[16,17,18].

Multi-domain uniform constrained simulation model's mainly strategy is to express every fields designing parameters, constraints conditions, and solving aims in a uniform statement equations. Because of product's expression often are hierarchy model, and MEMS' constraints relation model is also a part of product, so the uniform constrained simulation model also adopt hierarchy model like Fig1. According to classification of products, we divided MEMS constraints as product class constraints, components class constraints, and parts class constraints, and every class constraints have been divided constructive constraints, interface constraints, and associated constraints, and all the collects and relationship of the same class make up the constraints net. The constructive constraints express the MEMS's own characteristics, interface constraints express relationship between different fields, and associated constraints express the relationship between constructive constraints and interface constraints. 

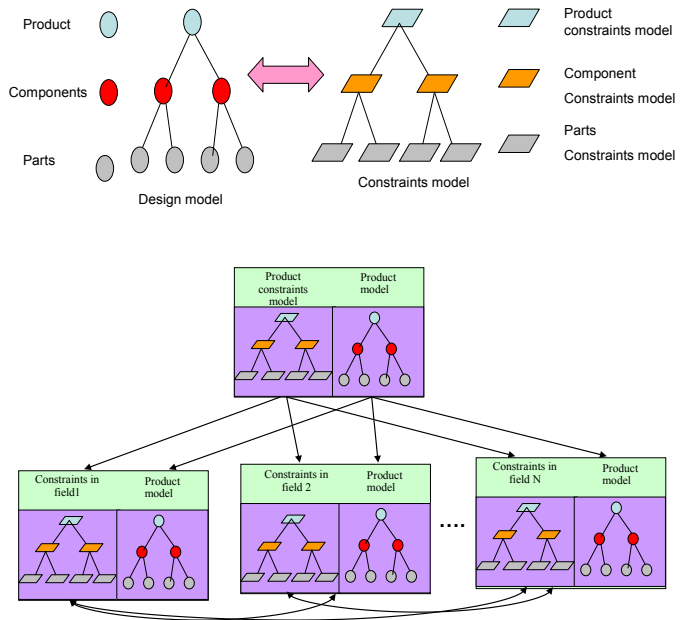

Fig 1 MEMS Product constraints relationship model

According to designing and analyzing of product should satisfy constraints, through grasp nature of different fields, we refine constraints as three elements: port, physical rule and element body, their relationship and expression are show as Fig2. The port was little circle of graph, the element bodies were linked by these ports and through object oriented and equation technology, make the constructive constraints, interface constraints, and associated constraints as a union expression. The constructed Micro-mechanical accelerometer united constrained model with modelica language was showing like figure 3.

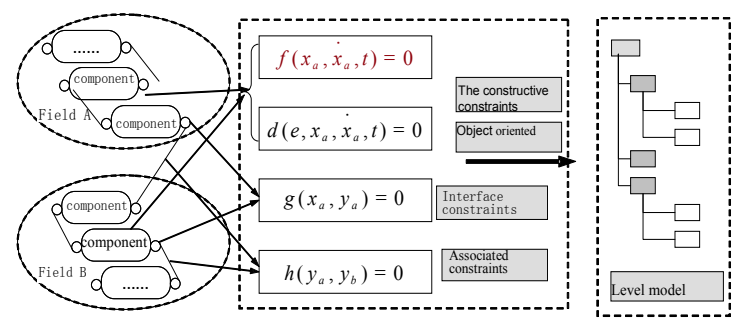

Fig 2 MEMS union constraints model

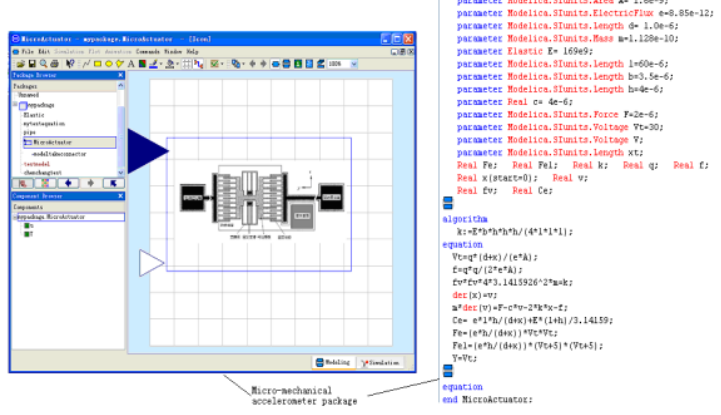

Fig 3 Micro-mechanical accelerometer united constrained model with modelica language
Exerting a harmonic force $F=F_{0} \sin (2 \pi f t)$ along the horizontal direction to the accelerometer, the amplitude is $F_{0}=1 \times 10^{-8} \mathrm{~N}$, the frequency is $f=11933 \mathrm{~Hz}$, taking the quality of the mass $m=4.58 \times 10^{-11} \mathrm{~kg}$, the damping coefficient is $c=1.76 \times 10^{-7} \mathrm{Ns} / \mathrm{m}$, stiffness coefficient of the beam is $k=0.2576 \mathrm{~N} / \mathrm{m}$, after simulate in Dymola for three millisecond, we can get the speed character istic curve as shown in figure 4, you can see the quality mass reach steady state quickly.

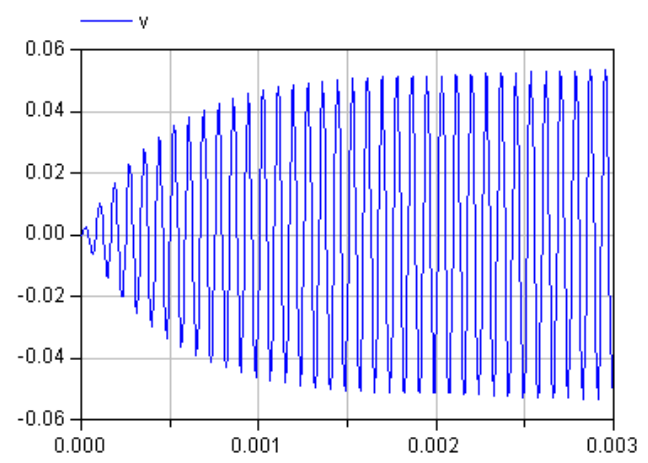

Fig.4 The speed curve of the accelerometer under harmonic external force

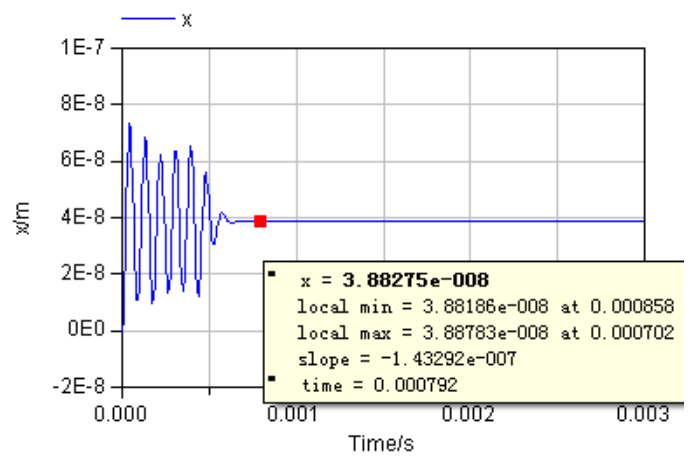

Fig.5 The displacement output curve under the constant force

By changing the form of the input force, we change the force to a constant force $F=1 \times 10^{-8} N$, change the Modelica procedure parameters $\mathrm{F}$ of figure 4 , the rest of the parameters are the same, simulating in the Dymola platform, then we can get the displacement output curve as shown in figure 5, we can see from the figure 6 the displacement of the accelerometer eventually tend to $\mathrm{x}=38.8275 \mathrm{~nm}$, almost the same to the theoretical value $\mathrm{x}=38.8198 \mathrm{~nm}$.

\section{IV . Conclusion}

The MEMS simulation model based on multi-domain unified constraint expression use a unified constrained model to describe the MEMS multi-domain optimization problem, which can embody the multi-field and hierarchical characteristics of MEMS, it has the unified constraint representation theory with the physical structure intrinsic and reusable characteristics. Before solving the situation, we 
should coordinate the coupling factors of each field, guide the optimal solution obtained under the unified constraint environment, improve the quality of solution, enhance the fault tolerance ability and improve the information exchange ability. Solving use the constraint mapping and structural reduction technology to achieve the solution of multi-field optimizations design model under a large scale constraints environment, and ultimately realize the solving theoretical system of MEMS unified constraint modeling and simulation optimization. Therefore, compared to other methods, multi-domain unified modeling and simulation optimization design method can better solve the MEMS simulation optimization problems.

\section{Acknowledgment}

The paper was support by the National Natural Science Foundation of China "MEMS Multi-fields Uniform Simulation Model Construction and Optimism Research" (61100101, 51275141 ), the paper was also supported by Key Discipline of The Ocean Techtronic Equipments Technology.

\section{References}

[1] Li Desheng: MEMS Technology and its Application.Haibin industrial university press. 2002

[2] L.S.Fan, Y.C.Tai, R.S.Muller.IC-processed electrostatic micromotor. Sensors and Actuators, 1989, 20 (10): 41-47

[3] Hu Wei,Hu Guoqing, Wei Xin and Xie Xiaozhu.MEMS CAD Research Development and the Present Situation. Piezoelectrics and Acoustooptics. 2010, 32 (4): 682-691

[4] Aluru N R, White J.Multi-level Newton method for static and fundamental frequency analysis of electromechanical systems.International Conference on Simulation of Semiconductor Processes and Devices, SISPAD, 1997: 125-128

[5] X. Rottenberg, P.Czarnecki, H.A.C.Tilmans, W.De Raedt. Multi-physics simulation and reliability analysis for RF-MEMS devices. $9^{\text {th }}$. Int. Conf. on Thermal, Mechanical and Multiphysics Simulation and Experiments in Micro-Electronics and Micro-Systems, EuroSimE 2008: 319-329

[6] Li Jun, Ying Ji and Zhou Liangxing. Based on the MEMS Static Micropump Modeling and Simulation. Journal of Engineering Design. 2009, 16 (1): 63-66

[7] Ji Kexing, Song Hongwei and Fan Xuejun. Residual Stress Analysis of High-temperature MEMS Sheer Stress Sensor. Mechanics and Practice. 2010, 32 (1): 60-63
[8] ROBERT L. EWING, Technology Road Map to Methodologies for Mixed-Signal System Design and simulation, Journal of VLSI Signal Processing. 1999, 20: 123-134

[9] Wang Yongquan, Chen Hualing, Peng Bei and Zhu Zicai. MEMS Node Modeling and its Application of Integrated Microsystem Design. Optical Precision Engineering. 2010, 18 (1): 198-203

[10] Jan E.Vandemeer, Nodal Design of Actuators and Sensors (NODAS), Technical Report, May 7, 1998, Carnegie Mellon University

[11] Q.Jing, T.Mukherjee and G.Fedder, Schematic-Based Lumped Parameterized Behavioral Modeling for Suspended MEMS, in Technical Digest of the ACM/IEEE International Conference on Computer Aided Design (ICCAD 02), San Jose, California, 2002: 367-373

[12] Yuan Weizheng, Niu Haobin, Chang Honglong and Ma Binghe. Cosimulation Prototype System of MEMS devices and Circuit. Optical Precision Engineering. 2010, 18 (10): 2206-2210

[13] Gou Peng, Liu Wei and Gui Weicheng. A Comparison of Approximation methods for Multidisciplinary Design Optimization of Ship Structures. Journal of Ship Mechanics. 1978, 11 (6): 913-922

[14] Lee J.Y, K.Kim. Geometric Reasoning for Knowledge-Based Design Using Graph Representation. Computer Aided Design. 1998, 28 (19): 831-841

[15] Mattsson SE, Elmqvist H, Otter M. Physical system modeling with Modelica. Control Engineering Practice. 1998, 6 (4): 501-510

[16] Zhou Xiangjun, Jin Shuang, Chen Yan, Lu Jun, Wang Hongjun and Liu Tianhu. Picking Manipulator Motion Control Modeling Based on the Modelica. Journal of System Simulation. 2009, 21 (18): 5882-5885

[17] Zhang Sheng, Cheng Wenke, Wang Hua and Tang Guojin. Ballistic Missile System Simulation Based on Modelica Language. Journal of Missiles and Guidance. 2010, 30 (5): 20-25

[18] Chen Qiongzhong, Meng Guang. The Modelica Modular Modeling Method of Switched Reluctance Motor. Motor and Control Applications. 2009, 36(12): 1-5

[19] Cui Jiuzheng, Sun Bo and Feng Qiang. Sensitivity Analysis of Factors Influencing MEMS Package Reliability. Prognostics and System Health Management Conference. Publication Year:2011, Page (s): 1-7

[20] A.Kohler, S.Reitz and P.Schneider. Sensitivity Analysis and Adaptive Multi-point Multi-moment Model Order Reduction in MEMS Design. Design, Test, Integration and Packaging of MEMS/MOEMS. Publication Year: 2011, Page (s): 64-71

[21] Cheng.J, Liao.S.J, Mohapatrab, R.N. and Vajravelu.K, Series Solutions of Nano Boundary Layer Flows by Means of the Homotopy Analysis Method. Journal of Mathmatical Analysis and Applications. 2008, 343 (1): 233-245

[22] Z.Li, B.Han. A Parameter Differential Regularization Algorithm for Solving Inverse Problems of GPR. Journal of Computational Information system. 2008, 4 (1): 407-412

[23] Gong Youping, Jin Tao and Tong Shuiguang. Application of Homotopy Method in the Reverse Engineering Constraint solving. Journal of Image and Graphics. 2007, 12 (4): 727-731 